\title{
An analytical model of crenulate shaped beaches
}

\author{
H.G. Wind \\ University of Twente, Dept. of Civil Engineering and Management, P.O. Box 217, 7500 AE Enschede, \\ Netherlands
}

Received 23 June 1993; accepted after revision 17 December 1993

\begin{abstract}
An analytical model for the development of crenulate shaped beaches is constructed. It is assumed that the shape of the crenulate shaped beach remains constant with time and expands with a rate according to a time function. Based upon a sediment balance, a simplified model of wave diffraction and refraction and a nearshore current model an expression for the shape function and the time function of crenulate shaped beaches is derived. It is shown that in the diffraction zone the time function should follow a $t^{1 / 3}$ law, while for the refraction zone the well known $t^{1 / 2}$ law is found. This implies that the evolution of a crenulate beach in the diffraction zone should initially be faster and on the long term slower than is found in refraction zones. The hypothesis is verified using available data. The resulting shape function of crenulate shaped beaches is expressed in terms of the diffracted wave field.
\end{abstract}

\section{Introduction}

Crenulate shaped beaches present on the one hand the sheer beauty of the morphological architecture of nature and on the other hand an area of redistribution or loss of sediment. The cause of the change of the water line is often formed by a natural or artificial fixed point which affects both the incoming wave field and the related wave driven sediment transport. For the resulting curved beaches various names have been suggested, such as headland-bay beaches (Yasso, 1965), crenulate shaped beaches (Silvester and Ko, 1972) and spiral beaches (Le Blond, 1972). The importance of crenulate shaped beaches for coastal engineering is related to their stabilizing effect on the shoreline as can be observed near headlands and nearshore breakwaters (Suh and Dalrymple, 1987; Hsu et al., 1989).

Characteristic for the type of sedimentary beaches, which will be considered in this paper, is the presence of at least one natural or artificial fixed point, leading to diffraction of the incoming wave field. Furthermore the resulting beach shape in the diffraction zone is expanding at a rate which decreases with time. Finally outside the diffraction zone the 
crenulate shaped beach matches the receding waterline approached by the refracted wave field.

Various mathematical shapes have been proposed to fit crenulate shaped beaches. Mashima (1961) used elliptic shapes. Yasso (1965) found that at least part of the crenulate shaped beaches followed a logarithmic spiral. Silvester developed this approach in various papers. In Hsu et al. (1989) drawbacks of the logarithmic spiral approach are mentioned, such as difficulties in defining the downcoast limits of bayed beaches and of the position of the pole. Hsu suggests that the logarithmic spiral should be replaced by some new relationships.

Laboratory studies and field data on bays with one or two fixed points are found for instance in Vichetpan (1971), Suh and Dalrymple (1987) and Hsu et al. (1989). The present analysis is restricted to beaches with only one fixed point. Various mathematical model studies of crenulate shaped beaches have been presented, by e.g. Le Blond (1972), Kraus (1983) and Hanson (1987). In these studies the nearshore region is represented as a one-dimensional shoreline model and the wave field contains both refraction and diffraction effects. In most of these studies the interaction between nearshore wavefield and the shape of the crenulate shaped beaches is emphasized and secondly the decreasing rate of expansion of the beach is highlighted. Mathematical solutions of both aspects are lacking.

In this paper an onset will be given to a formulation which will yield an expression for the expansion of crenulate beaches with time, as well as a solution for the shape of crenulate shaped beaches related to the characteristics of the nearshore wavefield. Particularly in the area of wave refraction and diffraction a large body of knowledge has been developed which could be used in the proposed approach.

\section{The conceptual model}

In the literature general descriptions can be found of both the dynamic evolution and the equilibrium stage of crenulate shaped beaches. Hsu et al. (1989) describe the equilibrium stage and give empirical relations. The dynamic stage follows from experiments by Vichetpan (1971) and is described in Silvester and Ko (1972) and Le Blond (1979). A conceptual model for the description of the evolution of crenulate shaped beaches will be derived from the work of Vichetpan (1971) and it will be shown that in the long term this conceptual model approaches the static model presented by Hsu et al. (1989).

\subsection{Static crenulate shaped beaches}

Hsu et al. (1989) has described stable crenulate shaped beaches as follows. "The bays may be in dynamic equilibrium with continual sediment supply or in static equilibrium when further littoral drift is taking place. Such a bay in static equilibrium has a near tangential straight segment downcoast, followed by a logarithmic-spiral curve, and then connected to an almost circular section behind the headland upcoast. In this stable condition, the tangential section is parallel to wave crests approaching the coast from offshore and the incoming waves will refract and diffract into the bay and break simultaneously along the whole 
periphery'. Along these equilibrium beaches the net longshore sediment transport is nil and the beach changes are dominated by temporal changes and offshore sediment transport.

\subsection{Dynamic crenulate shaped beaches}

An example of the dynamic evolution of a crenulate shaped beach is found in the experiments by Vichetpan (1971). These experiments started with a straight sandy beach and obliquely incident waves, with an angle of incidence between the incoming wave direction and the original shoreline of $(\pi / 2-\lambda)$. In the description of these experiments in Silvester (1974) the same three elements of the static beach are found: a circular sector, a logarithmic spiral and a straight beach section. The growth towards equilibrium was achieved by the tangential alignment changing from the original headland chord to a direction parallel to the crests of the incoming waves. This tangent commences at the downcoast headland and grows steadily in length as equilibrium is approached.

\subsection{The conceptual framework}

The hypothesis which can be derived from the experiments of Vichetpan and of the previous descriptions is that crenulate shaped beaches are centred around a pole and evolve in time in a more or less constant shape. In Fig. 1 the pole is located in point $\mathrm{A}$ and the

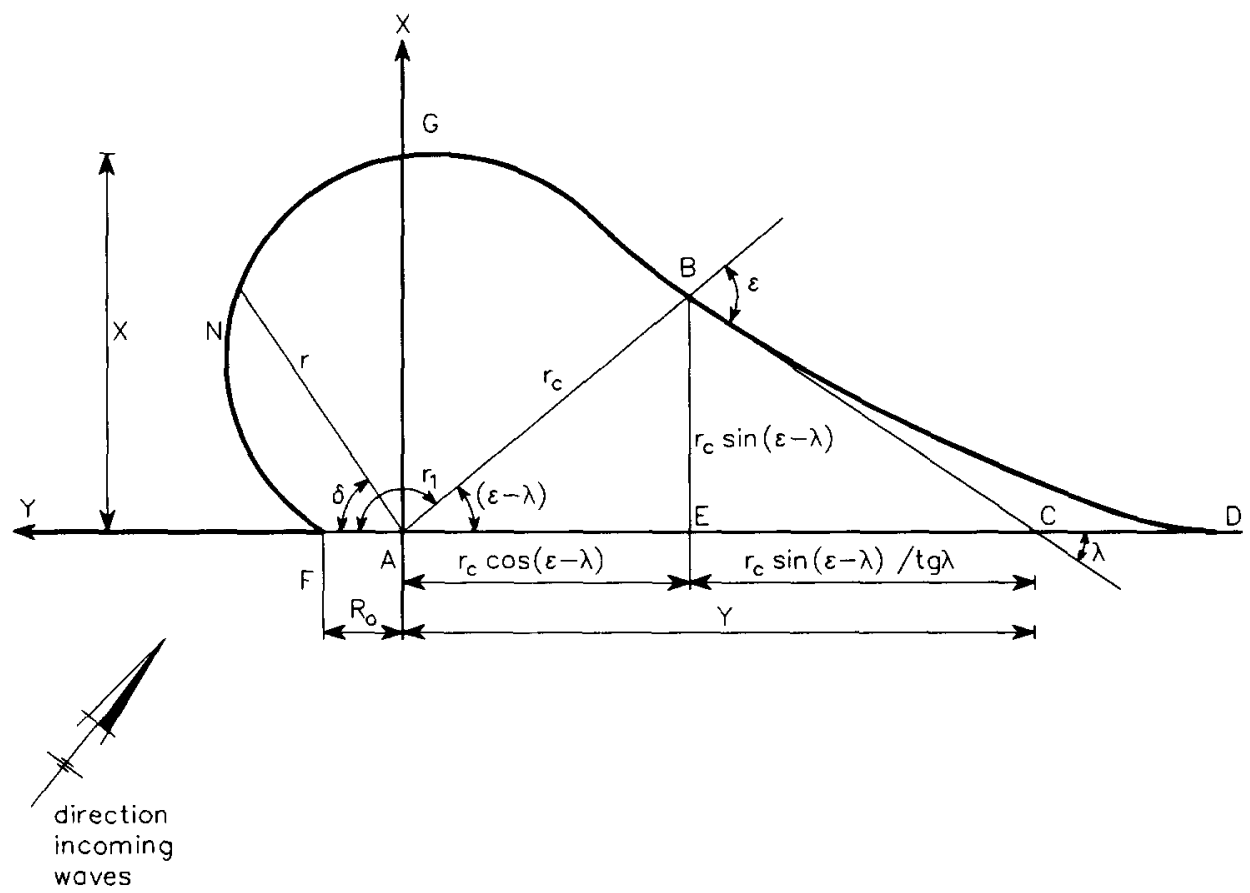

Fig. 1. Definitions. 
position of the shoreline is described by the radius $r$, the angle $\delta$ and the time $t$. Evolution of the shoreline with a constant shape implies that the shoreline might be described as:

$$
r(\delta, t)=r_{\mathrm{o}} f(\delta) e(t)
$$

where $r_{\mathrm{o}}$ is a constant, $f(\delta)$ is the shape function and $e(t)$ represents the evolution of the beach in time $t$.

As far as the straight beach section is concerned initially the suggestion of Silvester and Ko (1972) will be followed and the straight beach section will be kept straight. However as the angle of wave incidence along the original beach line $\mathrm{CD}$ in Fig. 1 is larger than along section $\mathrm{BC}$, it is expected that in the dynamic stage the sediment transport rate along $\mathrm{CD}$ will be larger than along $\mathrm{BC}$. This will lead to a discontinuity in the sediment transport rate at point $C$ and hence additional erosion is expected from $B C$ towards the line $B D$. For modelling of that part of the beach the approaches presented by Pelnard-Considère (1956) or Bakker (1968) can be used. Obviously in the long term the erosion of the section BD will continue, leading to a shift of point $B$ in the direction of point $G$ and a consecutive rotation of section $\mathrm{BD}$ until the sediment transport rate is equal to zero as is described by the static model of Hsu et al. (1989).

\section{Theoretical framework}

The theoretical framework consists of the continuity equation and an expression for the sediment transport rate. Because of the complex wave refraction/diffraction and the resulting flow field in the vicinity of the spiral beach, the various relevant physical processes first will be represented by general functional relations. This will lead to one solution for the time function $e(t)$ and a class of solutions for the shape function $f(\delta)$. By selecting specific representations for the relevant physical processes the resulting shape of the crenulate shaped beach can be derived from the general solution.

Because of the nature of the problem, the framework will be expressed in polar coordinates.

\subsection{The contunuity equation}

The continuity equation for sediment in polar coordinates reads:

$$
\frac{\partial S}{\partial \delta}+h r \frac{\partial r}{\partial t}=0
$$

where $S$ represents the sediment transport rate, $r$ is the radius and $\delta$ is the angle between the $y$-axis and the radius $r$. The erosion rate or accretion rate $\partial S / \partial \delta$ is assumed to be uniformly distributed over a depth $h$, which is assumed to be constant along the beach. 


\subsection{Sediment transport formulation}

In Longuet-Higgins $(1970,1971)$ the longshore sediment transport rate is related to the longshore gradient in wave action $\partial S_{x y} / \partial y$. In terms of significant wave height conditions near the surf zone the sediment transport rate can be expressed as:

$$
S=A H_{s}^{2} n_{\mathrm{s}} c_{\mathrm{s}} k(\beta)
$$

where $A$ is a constant. The significant surf conditions are expressed by the wave height $H_{s}$, the wave celerity $c_{\mathrm{s}}$ and $n_{\mathrm{s}}$ : the ratio of the wave celerity and the group velocity of the waves. The geometrical part of the driving force is expressed by $k(\beta)$ and is explained below.

\subsection{The geometrical part of the driving force $k(\beta)$}

The function $k(\beta)$ represents the geometrical part of the driving force of the longshore currents. This function is related to the angle $\beta$ between wave crest and the shoreline. Along a straight and uniform beach $k(\beta)$ can be represented by:

$$
k(\beta)=\frac{1}{2} \sin 2 \beta(\delta)
$$

Because of the complex wave and current conditions in the diffraction zone alternative forms of $k(\beta)$ can be envisaged; therefore $k(\beta)$ will retained in a generalized form.

\subsection{Wave characteristics and the functions $b(\delta)$ and $l(\delta)$}

A wave field, reaching the crenulate shaped beach, will strongly diffract initially in the vicinity of A-F (see Fig. 1) and continue its path with a diminishing effect of diffraction and an increasing effect of refraction and shoaling. Characteristic for a developing spiral beach is that the wave action, which in the long term will remain more or less constant, is distributed over a continuously increasing length of beach. This will affect both the shape $f(\delta)$ and the rate of change of the crenulate shaped beach $e(t)$.

In order to analyse this effect of a constant flux of wave momentum on an increasing length of beach the following simple model is constructed. It is assumed that diffraction effects are restricted to area bounded by an arc with radius $r_{1}$ (see Fig. 1). The significant wave height distribution along this arch will be described by:

$$
H\left(r_{1}, \delta\right)=H_{1} b(\delta)
$$

where $H_{1}$ is the significant wave height and $b(\delta)$ denotes the unit wave height distribution along $r_{1}$. Outside the diffraction zone the wave field will match the refracted wave field. An estimate of $b(\delta)$ can be obtained for instance from the analytical solution of wave diffraction on water of uniform depth. References and formulation can be found in Berkhoff (1976). Only for demonstration purposes the approximation $b(\delta)=\delta^{m}$ will be used, where $m$ is is a constant.

Along with $r_{1}$ also the product $n c$ must be specified, where $c$ is the wave celerity and $n$ 
is the ratio between group velocity and wave celerity. Similarly as for the wave height, the product $n c$ on $r_{1}$ will be specified by a constant value $n_{1} c_{1}$ and a function $l(\delta)$ or:

$$
n c\left(r_{1}, \delta\right)=n_{1} c_{1} l(\delta)
$$

In the area between $r_{1}$ and the surfzone shoaling is regarded to be a dominant effect while refraction will initially be neglected. This implies that the wave direction is assumed to remain parallel to the rays radiating from pole A. In order to account properly for wave refraction, the interaction of the wave field with the unknown curvi-linear bottom topography should be taken into account. This is well beyond the scope of the present paper. As a first step the effect of refraction could be accounted for in the function $k(\beta)$.

If dissipation of wave momentum is neglected in the region between $r_{1}$ and the surfzone, the wave height distribution along the surfzone over an angle $\mathrm{d} \delta$ follows the continuity of flux of momentum:

$$
\frac{1}{8} \rho g H_{1}^{2} b^{2}(\delta) n_{1} c_{1} l(\delta) r_{1} \mathrm{~d} \delta=\frac{1}{8} \rho g H_{\mathrm{s}}^{2} n_{\mathrm{s}} c_{\mathrm{s}} r_{\mathrm{s}} \mathrm{d} \delta
$$

where $\rho$ is the fluid density and $g$ represents the acceleration of gravity. The indices 1 and $\mathrm{s}$ refer to to the arch $r_{1}$ and the surfzone respectively.

\subsection{Evaluation of a crenulate shaped beach}

Based upon the simple model, which has been outlined in equation 2 through 7 the following equation for the evolution of a crenulate shaped beach, expressed in a shape function $f(\delta)$ and a time function $e(t)$ (see Eq. 1), can be obtained from Eq. 2:

$$
\frac{\partial}{\partial \delta}\left\{f^{-1} b^{2} k l\right\}+h r_{\mathrm{o}}^{3} f^{2} e^{2} \frac{\mathrm{d} e}{\mathrm{~d} t} /\left(B_{1} r_{1}\right)=0
$$

where $B_{1}$ equals:

$$
B_{1}=A H_{1}^{2} n_{1} c_{1}
$$

In the derivation of Eq. 8 use has been made of Eq. 1 and it has been assumed that the position of the surfzone $r_{\mathrm{s}}$ can be approximated by the position of the line $r$.

In section 4 an expression for the time function $e(t)$ will be derived from Eq. 8, while in chapter five a general solution for the shape function $f$ will be obtained.

\section{The time function $e(t)$ and $r_{\mathrm{o}}$}

In Eq. 8 an expression is presented, containing both $f(\delta)$ and $e(t)$. If Eq. 8 is indeed a dimensionless equation for $f(\delta)$ then the second term of Eq. 8, apart from $f^{2}$, has to be a constant, say $C$. For convenience $C$ is split into two factors which are defined by Eqs. 10 and 11: 


$$
\begin{aligned}
& e^{2} \frac{\mathrm{d} e}{\mathrm{~d} t}=\frac{A_{\mathrm{o}}}{3} \\
& r_{\mathrm{o}}^{3}=3 B_{1} r_{1} /\left(A_{\mathrm{o}} h\right)
\end{aligned}
$$

where $A_{\mathrm{o}}$ is a constant, which remains to be determined. For the solution of Eq. 10 the following initial and scaling conditions are imposed:

$$
\begin{array}{ll}
t=0, & e(0)=0 \\
t=T, & e(T)=1
\end{array}
$$

where the scaling condition 13 defines the time scale $T$ where the crenulate shaped beach has expanded to its unit size and can be described by:

$$
r=r_{\mathrm{o}} f(\delta)
$$

The time scale $T$ replaces the constant $A_{\mathrm{o}}$ as will be shown in Eq. 16.

The solution of Eq. 10, under the conditions 12 and 13 yields:

$$
e(t)=\left(\frac{t}{T}\right)^{1 / 3}
$$

in which

$$
T=\frac{1}{A_{\mathrm{o}}}
$$

The time scale 15 of a crenulate shaped beach in the diffraction zone may be compared with the time scale of erosion or sedimentation of a longshore driven beach evolution as described by the diffusion equation (Pelnard-Considère, 1956). The time scale in that case is $\sqrt{4 a t}$, where $a$ is a constant. This implies that the early development of beaches in the diffraction zone relatively is faster, whereas on the long term it is relatively slower than according to the well-known shoreline theory.

Based upon Eqs. 15 and 16 the description of the crenulate shaped beach as indicated in Eq. 1 becomes:

$$
r=r_{\mathrm{o}} f(\delta)\left(\frac{t}{T}\right)^{1 / 3}
$$

Eq. 17 can be used to calculate the time-variation during evolution of the crenulate shaped bay. The function $r_{\mathrm{o}} f(\delta)$ denotes a unit shape function of the bay. In order to verify Eq. 17 the data of Vichetpan (1971) has been used. For this verification the following procedure was chosen. First the value of $r_{\mathrm{o}} f(\delta)$, which for a fixed value of $\delta$ should be a constant, has been calculated for the four cross-sections shown in Fig. 2. Next the data has been plotted in a graph with axis $t^{1 / 3}$ and $r /\left(r_{\mathrm{o}} f(\delta)\right)$. If $r_{\mathrm{o}} f(\delta)$ is indeed a constant for a fixed value of $\delta$ and if a $t^{1 / 3}$ law is followed, than all the experimental data should fall along the diagonal.

The value of the constant $r_{\mathrm{o}} f(\delta)$ has been calculated from the experimental by applying to each cross-section: 


$$
r_{\mathrm{o}} f(\delta)=\sum r_{\mathrm{i}} / \sum t_{\mathrm{i}}^{1 / 3}
$$

The resulting values are given in Table 1 .

The scaling constant $T$ in the experiment of Vichetpan has been set at $T=1$ hour. Using Eq. 17 and the constants in Table 1, the data of the experiment shown in Fig. 2 has been plotted in Fig. 3. Taking into account the difficulties of long term experiments in morphological models, the agreement between theory and data is fair. This implies that indeed $r_{\mathrm{o}} f(\delta)$ is a constant for a fixed value of $\delta$ and that the evolution of a crenulate shaped beach follows a $t^{1 / 3}$ law.

Substitution of Eqs. 10,11 and 16 into Eq. 8 yields the following differential equation for the shape function $f(\delta)$ :

$$
\frac{\mathrm{d}}{\mathrm{d} \delta}\left\{f^{-1} b^{2} k l\right\}+C f^{2}=0
$$

For the solution of $f(\delta)$ from Eq. 19 , the functions $b, k$ and $l$ must be specified, together

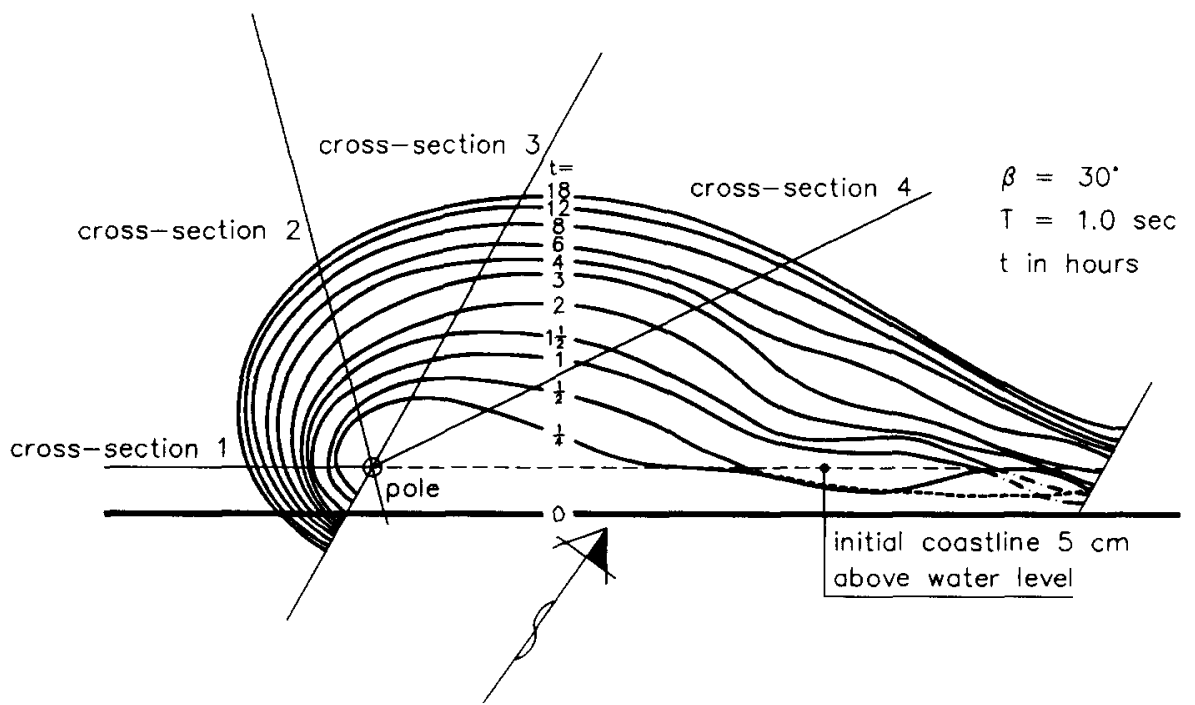

Fig. 2. Experimental data of the evolution of a spiral beach (after Vichetpan, 1971).

Table 1

The values of $r_{\mathrm{o}} f(\delta)$ for four cross-sections of the data of Vichetpan (1971)

\begin{tabular}{lr}
\hline Section & $r_{\mathrm{o}} f(\delta)$ \\
\hline 1 & 7.25 \\
2 & 12.17 \\
3 & 18.42 \\
4 & 30.22 \\
\hline
\end{tabular}




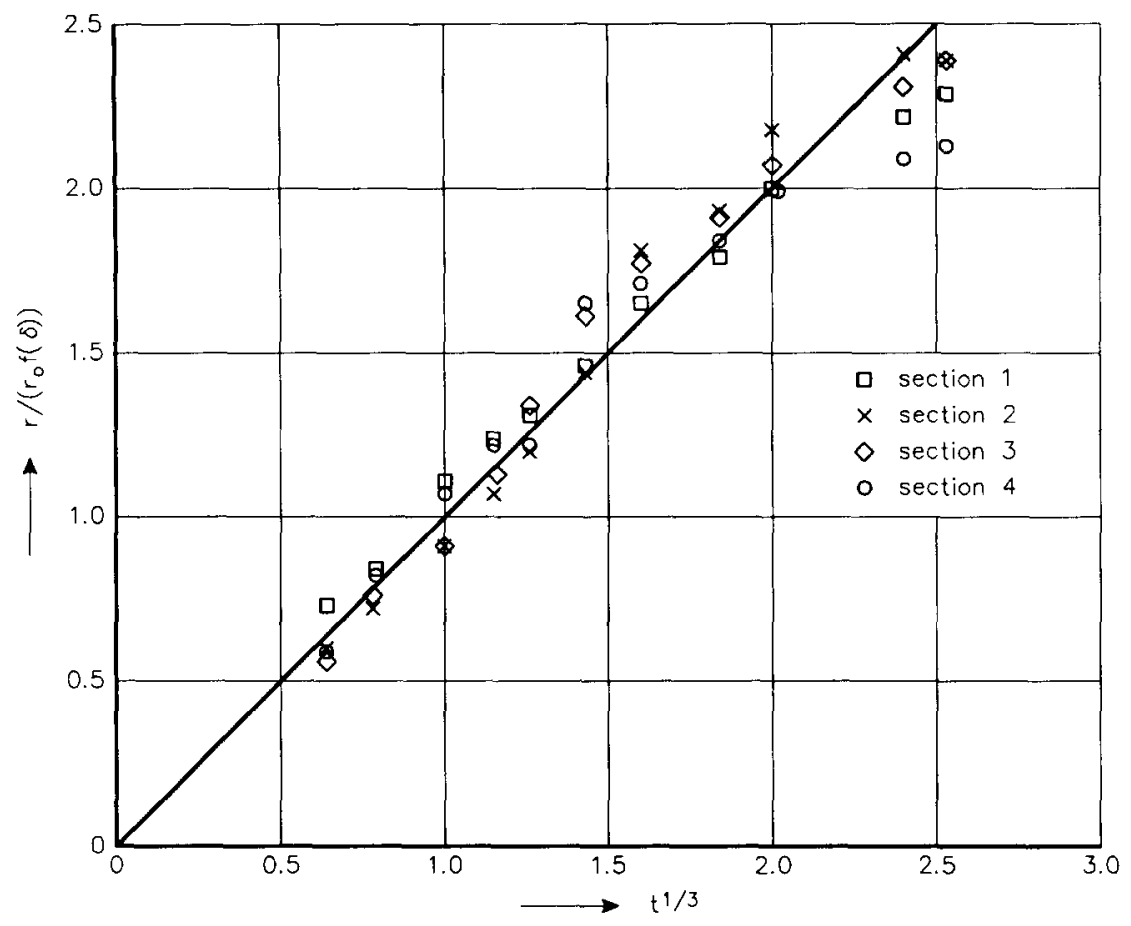

Fig. 3. Experimental verification of the $t^{1 / 3}$ time low for spiral beaches.

with a boundary condition. In the next section a general solution of $f(\delta)$ is derived together with some empirical approximations.

\section{Solutions of the shape function $f$}

The general solution of shape function $f$ follows from Eq. 19 and can be expressed as follows:

$$
f(\delta)=-b^{2} k l\left\{3 C \int_{0}^{\delta}\left(b^{2} k l\right)^{2} \mathrm{~d} \delta-c\right\}^{-1 / 3}
$$

In Eq. 20 the shape function $f(\delta)$ is expressed in functions representing the diffracted wave field: the function for the wave height distribution $b$, the function for the group velocity $l$ and the geometrical part for the driving force $k$.

Inversely, if the shape of the spiral beach is known, it follows from Eq. 19 that the representation of the wave field $b^{2} k l$ should satisfy:

$$
b^{2} k l=-f \int_{0}^{\delta} C f^{2} \mathrm{~d} \delta+c
$$


Introduction of representations for $b^{2}, k$ and $l$ in Eq. 20 leading to solutions of $f(\delta)$ is rather straightforward. The present analysis will be restricted to a simple example.

\subsection{Some examples off}

Suppose that the following representations for $k, l$ and $b$ are introduced:

$$
\begin{array}{ll}
k=\sin 2 \beta & (\beta=\text { constant }) \\
b=\delta^{m} & (m=\text { constant }) \\
l=1 & (1=\text { constant })
\end{array}
$$

Disregarding the integration constant in Eq. 20 the resulting shape function of $f$ is:

$$
f(\delta)=c \delta^{(2 m-1) / 3}
$$

where:

$$
c=-\sin 2 \beta\left\{3 C \sin ^{2} 2 \beta /(4 m+1)\right\}^{-1 / 3} \quad(m \neq-1 / 4)
$$

The solution of $f$ in 25 covers a wide range of spiral beaches. For $m=-1$ the hyperbolic spiral is obtained $(f \delta=$ constant). The spiral of Archimedes follows for $m=-1$ $(f=\delta \cdot$ constant $)$. For the Lituus, defined as $f^{2} \cdot \delta=$ constant, $m$ should equal $-1 / 4$. This value is excluded in Eqs. 25 and 26. Obviously the selection of an appropriate value of $m$ is determined by a realistic representation of the wave field in the diffraction zone $b=\delta^{m}$. The logarithmic spiral

$$
f=\mathrm{e}^{-\delta \operatorname{cotg} \epsilon}
$$

where $\epsilon$ is a constant which can be derived from Eq. 21 by neglecting the constant $c$, taking $k$ and $l$ constant and representing the unit wave height distribution in the diffraction zone by:

$$
b=\left\{-\mathrm{e}^{\delta \operatorname{cotg} \epsilon} \frac{C}{2 k \operatorname{cotg} \epsilon}\left(\mathrm{e}^{2 \delta \operatorname{cotg} \epsilon}-1\right)\right\}^{1 / 2}
$$

\section{Discussion}

In the one-dimensional description of erosion and sedimentation of sandy beaches a $t^{1 / 2}$ trend is found. For crenulate shaped beaches both theory and data seem to point towards a $t^{1 / 3}$ trend. This implies that initially the evolution of spiral beaches is faster and on the long term is slower than in case of one-dimensional nearshore erosion caused by refraction only. As this difference is caused by the fact that in a diffraction zone constant influx of wave momentum is to be spread over a continuously increasing length of beach, it is expected this conclusion is firmly founded. Including additional processes which will lead to a redistribution of wave momentum along the spiral beach is therefore expected to change this conclusion marginally.

The study of the shape function $f(\delta)$ has led to Eq. 19 with the examination of this 
equation and the resulting shape functions along the preliminary start has been made. The range of spiral beaches, resulting from $b=\delta^{m}$ is only partially realistic, because of the required diffracted wave field. The study of more realistic shape functions requires the input of appropriate functions for $b, k$ and $l$. This is an area of further research together with a survey for additional site information both on the shape of the spiral beach and the related input functions.

\section{Acknowledgement}

The author acknowledges the encouragement of $\mathrm{dr}$. H. de Vriend to publish this work and his critical comments on the initial paper. Furthermore the author acknowledges the permission of Delft Hydraulics to publish this research which in part has been executed while serving the laboratory.

\section{References}

Bakker, W.T., 1968. A mathematical theory about sandwaves and its application on the Dutch Wadden Isle of Vlieland. Shore Beach, 36(2): 4-14.

Berkhoff, J.C.W., 1976. Mathematical Models for Simple Harmonic Linear Water Waves. Publication 163, Delft Hydraulics.

Hanson, H., 1987. Genesis: a generalized shoreline change numerical model for engineering use. Ph.D. Thesis, Lund University, Report No. 1007.

Hsu, J.R.C., Silvester, R. and Xia, Y.M., 1989. Generalities on static equilibrium bays. Coastal Eng., 12: 353369.

Kraus, M.C., 1983. Application of a shoreline prediction model. In: J.R. Weggel (Editor), Proc. of Coastal Structures, Arlington, VA, Vol. 2, pp. 632-645.

Le Blond, P.H., 1972. On the formation of spiral beaches. In: Proc. 13th Int. Conf. Coastal Eng., Vancouver, B.C., pp. 1331-1345.

Le Blond, P.H., 1979. An explanation of the logarithmic spiral plan shape of headland bay beaches. J. Sed. Petrol., 49: $1093-1100$.

Longuet-Higgins, M.S., 1970. Longshore currents generated by obliquely incident sea waves. J. Geophys. Res., 75(33): 6778-6801.

Longuet-Higgins, M.S., 1971. Recent progress in the study of longshore currents in waves on beaches and resulting sediment transport. University of Wisconsin, Madison, USA Publ. 28, pp. 203-248.

Mashima, Y., 1961. Stable configuration of coastline. Coastal Eng. Jpn., 4: 47-59.

Pelnard-Considère, R., 1956. Essai de théorie de l'évolution des formes de rivage en plages de sable et de galets. In: IVèmes Journées de l'Hydraulique, Paris, pp. 74-1, 74-4.

Silvester, R. and Ko, S., 1972. Use of crenulate shaped bays to stabilize coasts. In: Proc. 13th Conf. Coastal Eng., pp. 1347-1365.

Silvester, R., 1974. Coastal Engineering II. Elsevier, Amsterdam.

Suh, K.S.M. and Dalrymple, R.A., 1987. Offshore breakwaters in laboratory and field. J. Waterw. Port Coastal Ocean Eng., 113(2): 105-121.

Vichetpan, M., 1971. Development of crenulate shaped bays. Thesis No. 280, Asian Inst. Technology, Bangkok. Yasso, W.E., 1965. Plan geometry of headland bay beaches. J. Geol., 73: 702-714. 